\title{
Application of FTIR spectroscopy for the determination of virgin coconut oil in binary mixtures with olive oil and palm oil.
}

\begin{abstract}
Rapid Fourier transform infrared (FTIR) spectroscopy combined with attenuated total reflectance (ATR) was applied for quantitative analysis of virgin coconut oil (VCO) in binary mixtures with olive oil (OO) and palm oil (PO). The spectral bands correlated with VCO, OO, $\mathrm{PO}$; blends of VCO and OO; VCO and PO were scanned, interpreted, and identified. Two multivariate calibration methods, partial least square (PLS) and principal component regression (PCR), were used to construct the calibration models that correlate between actual and FTIR-predicted values of VCO contents in the mixtures at the FTIR spectral frequencies of 1,120-1,105 and 965-960 $\mathrm{cm}-1$. The calibration models obtained were cross validated using the "leave one out" method. PLS at these frequencies showed the best calibration model, in terms of the highest coefficient of determination (R 2) and the lowest of root mean standard error of calibration (RMSEC) with R $2=0.9992$ and RMSEC $=0.756$, respectively, for VCO in mixture with OO. Meanwhile, the R 2 and RMSEC values obtained for VCO in mixture with PO were 0.9996 and 0.494 , respectively. In general, FTIR spectroscopy serves as a suitable technique for determination of VCO in mixture with the other oils.
\end{abstract}

Keyword: FTIR; Virgin coconut oil, Binary mixture; Parital elast square; Principal component regression. 